\title{
Sentinel Node Navigation Surgery in Patients with Early Gastric Cancer
}

\author{
Hiroya Takeuchi Yuko Kitagawa \\ Department of Surgery, Keio University School of Medicine, Tokyo, Japan
}

\section{Key Words}

Early gastric cancer $\cdot$ Quality of life · Sentinel node

\begin{abstract}
Clinical application of sentinel node (SN) mapping in patients with early gastric cancer has been a controversial issue for years. However, a recent meta-analysis and a prospective multicenter trial of SN mapping for early gastric cancer have shown acceptable $\mathrm{SN}$ detection rates and accuracy of determination of lymph node status. A dual-tracer method that utilizes radioactive colloids and blue dyes is currently considered the most reliable method for the stable detection of SNs in patients with early gastric cancer. However, new technologies such as indocyanine green infrared or fluorescence imaging might revolutionize the SN mapping procedures in gastric cancer. For early-stage gastric cancer such as CT1NO, for which a better prognosis can be potentially achieved through conventional surgical approaches, the establishment of individualized, minimally invasive treatments that may retain the patients' quality of life should be the next surgical challenge. Although there are many unresolved technical issues, laparoscopic SN biopsy with laparoscopic minimized gastrectomy or endoscopic mucosal resection/ endoscopic submucosal dissection could be a promising strategy to achieve this goal.

Copyright $\odot 2013$ S. Karger AG, Basel
\end{abstract}

\section{KARGER}

E-Mail karger@karger.com

www.karger.com/dsu

\section{Introduction}

Sentinel nodes (SNs) are defined as the first draining lymph nodes from the primary site of a tumor [1], and they are thought to be the first possible site of micrometastasis along the route of lymphatic drainage from the primary lesion (fig. 1). Therefore, the pathological status of SNs can theoretically predict the status of all regional lymph nodes. If SNs are recognizable and negative for cancer metastasis, unnecessary radical lymph node dissection might be avoided. The SN hypothesis is applied to patients at relatively high risk of lymph node metastasis, which is based on the characteristics of the primary tumor, but having clinically undetectable regional metastatic nodes as confirmed by preoperative diagnostic imaging. A more efficient and accurate diagnosis of lymph node metastasis along with adequate prognostic information can be obtained from a small number of lymph nodes through intraoperative SN mapping and biopsy $[1,2]$. SN navigation surgery is defined as a novel, minimally invasive surgery based on SN mapping and the SN-targeted diagnosis of nodal metastasis. This surgery can prevent unnecessary lymph node dissection, thus preventing the associated complications and improving the patient's quality of life (QOL).

SN mapping and biopsy were first applied to melanoma and breast cancer patients and were subsequently ex- (c) 2013 S. Karger AG, Basel

0253-4886/13/0302-0104\$38.00/0
Hiroya Takeuchi, $\mathrm{MD}, \mathrm{PhD}$

Department of Surgery, Keio University School of Medicine

35 Shinanomachi, Shinjuku-ku

Tokyo 160-8582 (Japan)

E-Mail htakeuchi@a6.keio.jp 
tended to patients with many other solid tumors [1-3]. The SN concept has revolutionized the approach to the surgical staging of both melanoma and breast cancer. Applying this concept can yield benefits for patients by preventing various complications related to unnecessary prophylactic radical lymphadenectomy such as axillary lymph node dissection in breast cancer patients with negative $\mathrm{SN}$ for cancer metastasis.

Many patients with early gastric cancer are currently treated with advanced laparoscopic gastrectomy procedures, such as laparoscopy-assisted distal gastrectomy and laparoscopy-assisted total gastrectomy, not only in Japan but also in other countries [4-7]. Advanced laparoscopic gastrectomy contributes to both better aesthetics and early postoperative recovery [8]. However, patients' QOL is mainly affected by late-phase complications, such as dumping syndrome and body weight loss resulting from oral intake disturbance. Therefore, both minimal invasiveness for early-phase recovery and satisfactory late-phase function after gastric cancer surgery should be carefully considered in patients indicated for these procedures.

Although function-preserving surgery such as partial gastrectomy, segmental gastrectomy, and proximal gastrectomy, with limited stomach resection and lymph node dissection, helps in improving postoperative latephase function, a certain incidence of skip metastasis in the 2 nd or 3 rd compartment of the regional lymph nodes remains an obstacle to the wider application of these procedures. To overcome these issues, the concept of SN mapping is anticipated to become a novel diagnostic tool for the identification of clinically undetectable lymph node metastasis in patients with early gastric cancer.

The clinical application of SN mapping for early gastric cancer has been controversial for years. However, single institutional results, including ours and those from a recent multicenter trial of SN mapping for early gastric cancer, are considered acceptable in terms of the SN detection rate and accuracy of determination of lymph node status $[9,10]$. On the basis of these results, we are developing a novel, laparoscopic, minimally invasive gastrectomy technique combined with SN mapping.

\section{Surgical Techniques of Laparoscopic SN Mapping}

A dual-tracer method that utilizes radioactive colloids and blue dyes is currently considered the most reliable method for the stable detection of SNs in patients with early gastric cancer $[11,12]$. An accumulation of radioactive colloids facilitates the identification of SNs even in

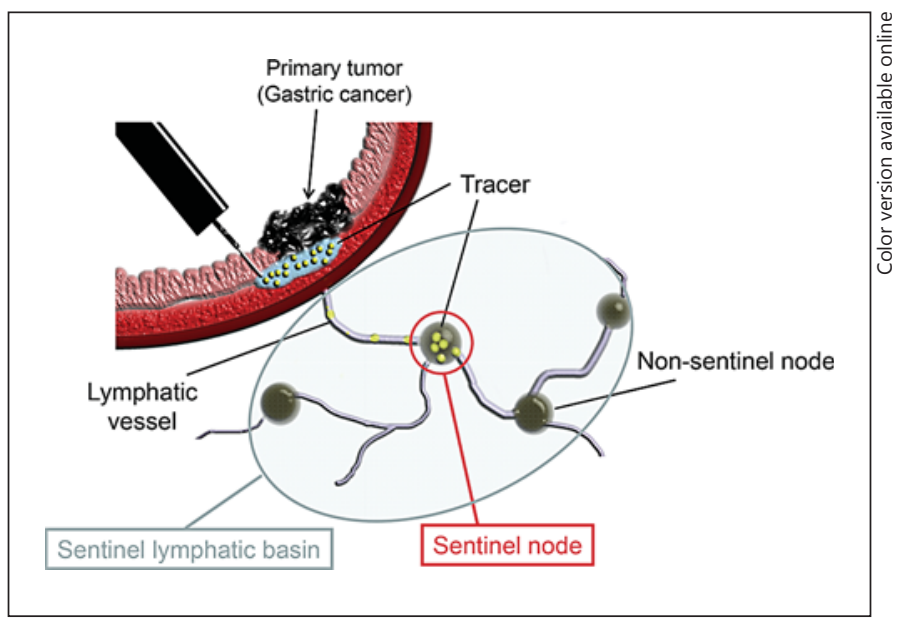

Fig. 1. Primary gastric cancer and $\mathrm{SN}$. The $\mathrm{SN}$ is defined as one or more lymph nodes that first receive lymphatic drainage from the primary tumor. For intraoperative SN mapping, tracers (blue dye and/or radioisotope-labeled colloid) are injected submucosally around the primary tumor site using endoscopy before surgery. Subsequently, the tracers pass through the afferent lymphatics, and stained or radioactive nodes are regarded as the SN.

resected specimens, and the blue dye is effective for intraoperative visualization of lymphatic flow, even during laparoscopic surgery. Technetium-99m tin colloid, technetium-99m sulfur colloid, and technetium-99m antimony sulfur colloid are preferentially used as radioactive tracers. Isosulfan blue, patent blue, and indocyanine green (ICG) are currently the preferred choices of dye tracers.

In general, patients with clinical T1 (or T2) tumors, primary lesions less than $4 \mathrm{~cm}$ in diameter, and clinical N0 gastric cancer undergo SN mapping and biopsy. In our procedures, $2.0 \mathrm{ml}(150 \mathrm{MBq})$ of technetium-99m tin colloid solution is injected the day before surgery into four quadrants of the submucosal layer of the primary tumor site using an endoscopic puncture needle. Endoscopic injections facilitate accurate tracer injection. Theoretically we would be able to identify any SNs even in unpredictable sites if we could inject the tracers appropriately into the submucosal layer surrounding the primary tumor using endoscopy. Therefore, there is no restriction of location of primary tumor in terms of the indication of SN mapping.

With its relatively large particle size, technetium-99m tin colloid accumulates in the SNs after local administration. According to our experience, it migrates into the SNs within $2 \mathrm{~h}$ and remains there for more than $20 \mathrm{~h}$ 
through phagocytosis by macrophages. Preoperative lymphoscintigraphy might be useful for the accurate detection of sentinel lymphatic basins at unexpected sites. However, we have previously demonstrated that only 71 (67\%) of 106 sentinel lymphatic basins were visualized during lymphoscintigraphy before gastrectomy [13]. In particular, higher body mass index affected unsuccessful SN detection by lymphoscintigraphy. Therefore, utility of the preoperative lymphoscintigraphy is still limited for SN mapping in early gastric cancer. Recently, some researchers have reported on the utility of single-photon emission computed tomography/computed tomography (SPECT/CT) for SN mapping in various malignancies [14]. They found that SPECT/CT for SN mapping brought precise localization with three-dimensional imaging. Application of SPECT/CT may improve the detection and the localization of SNs before gastric cancer surgery.

Similar to breast cancer and melanoma, cases with clinically apparent lymph node metastasis proven by preoperative imaging diagnosis such as CT scan and ultrasound would be excluded from the SN mapping because the purpose of this technique is to identify clinically undetectable lymph node involvement. Moreover, apparent metastatic lymph nodes occupied by massive cancer cells may interrupt the accurate detection of SNs because the influx of dye or radioactive tracers into lymph nodes could be blocked or altered by the obstruction of afferent lymphatic vessels. Therefore, routine preoperative imaging diagnoses to evaluate the lymph node involvement are also indispensable for successful SN mapping.

The first step of laparoscopic intraoperative SN detection performed at our institution involves the division of the gastrocolic ligament for the laparoscopic visualization of all possible directions of lymphatic flow from the primary site in the stomach [15-17]. Next, a blue dye (isosulfan blue or ICG) is injected under the guidance of intraoperative endoscopy, similar to the preoperative injection of the radioactive tracer. The blue dye is injected into four quadrants of the submucosal layer of the primary site using an endoscopic puncture needle. Blue lymphatic vessels and blue-stained nodes can be identified by laparoscopy within $15 \mathrm{~min}$ after the injection of the blue dye. The blue dye facilitates the visualization of lymphatic vessels despite several issues, such as the fast movement of the dye and blind sites in dense fat. Simultaneously, a hand-held gamma probe (GPS Navigator; RMD Instruments LLC, Watertown, Mass., USA) is used to locate the radioactive $\mathrm{SN}$. Intraoperative gamma probing is feasible in laparoscopic gastrectomy using a special gamma detec- tor introducible from trocar ports. Intraoperative sampling of SNs is not always easy because occasionally the radioactivity in $\mathrm{SNs}$ might have been hidden by the shinethrough effects of radioactivity in the primary tumor when they were in close proximity to the primary tumor. In general, however, radio-guided methods allow the confirmation of complete $\mathrm{SN}$ harvest by gamma probing. In contrast, the dye procedure enables real-time observation of lymphatic flow. We currently recommend a combination of blue dyes and radio-guided methods for systematic SN mapping in patients with gastric cancer.

For intraoperative SN sampling, the pick-up method is well established for the detection of melanoma and breast cancer. However, it is recommended that the clinical application of intraoperative SN sampling should include sentinel lymphatic basin dissection, which is a sort of focused lymph node dissection involving hot and blue nodes (fig. 1) $[18,19]$. The gastric lymphatic basins were considered to be divided in the following five directions along the main arteries: left gastric artery area, right gastric artery area, left gastroepiploic artery area, right gastroepiploic artery area, and posterior gastric artery area [20].

On the back table, the resected sentinel lymphatic basin is carefully investigated for hot nodes (SNs) using a hand-held gamma probe. Blue-stained or radioactive SNs identified in the resected specimens are carefully investigated using the gamma probe, and all SNs are sent for intraoperative pathological examination. After gastrectomy, the presence or absence of other radioactive SNs among the residual lymph nodes in the upper abdominal cavity is confirmed using the gamma probe, which is inserted from the trocar port or small abdominal wound.

ICG is known to have excitation and fluorescence wavelengths in the near-infrared range [21]. To date, some investigators have used infrared ray electronic endoscopy (IREE) to demonstrate the clinical utility of intraoperative ICG infrared imaging as a new tracer for laparoscopic SN biopsy (fig. 2) [22, 23]. Nimura et al. [24] previously reported a sensitivity of $100 \%$, specificity of $67 \%$, positive predictive value of $29 \%$, and negative predictive value of $100 \%$ for the detection of SNs using IREE (Olympus Optical, Tokyo, Japan) in 84 patients with cT1/ $\mathrm{T} 2$ and $\mathrm{cN} 0$ gastric cancer. IREE might be a useful tool to improve visualization of ICG-stained lymphatic vessels and SNs even in the fat tissues.

More recently, ICG fluorescence imaging has been developed as another promising novel technique for SN mapping [25]. Tajima et al. [26] reported on intraoperative ICG fluorescence imaging using a charge-coupled device camera (which filtered out light with wavelengths 


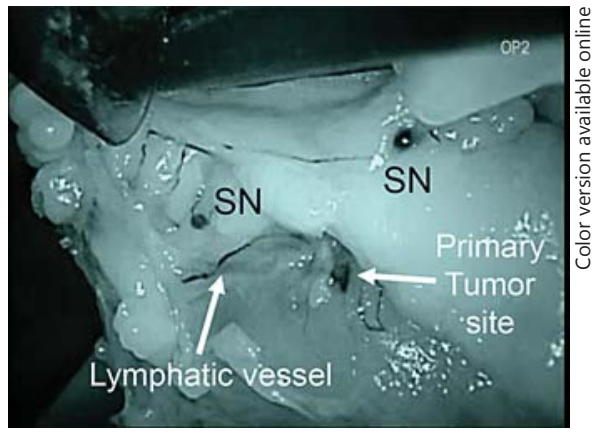

Fig. 2. ICG infrared imaging using IREE for laparoscopic SN napping. IREE can visualize even tiny SNs and thin lymphatics.

below $820 \mathrm{~nm}$ ) with a light-emitting diode (having a wavelength of $760 \mathrm{~nm}$; Hamamatsu Photonics, Hamamatsu, Japan) for intraoperative detection of $\mathrm{SN}$ in gastric cancer. SN could be clearly visualized by ICG fluorescence imaging compared to the naked eye. Moreover, the authors emphasized that SN identification using ICG fluorescence imaging was feasible even by preoperative ICG injection, e.g. 1-3 days before surgery, because the ICG particles binding with plasma proteins may spread out widely from the injection site through lymphatic vessels with time and accumulate in the SNs preoperatively. In addition, they reported it is also feasible in laparoscopyassisted gastrectomy introducible via a small laparotomy $[21,26]$.

In general, the number of $\mathrm{SN}$ identified by dye-guided methods including ICG infrared or fluorescence imaging is known to be relatively higher than that by radio-guided methods because the particle size of dyes is markedly smaller than that of radioactive colloids [16]. Therefore, SNs identified by dye-guided methods might contain not only SN but also the 2nd or 3rd compartment of regional lymph nodes. Further studies would be needed to evaluate the clinical efficacy of ICG infrared or fluorescence imaging and to compare those with radio-guided methods in prospective studies. These new technologies might revolutionize the SN mapping procedures not only in gastric cancer but also in many other solid tumors.

\section{Results of Intraoperative SN Mapping for Gastric Cancer}

To date, a number of single institutional studies have demonstrated acceptable outcomes of SN mapping for early gastric cancer in terms of the $\mathrm{SN}$ detection rate

SN Navigation Surgery for Early Gastric Cancer
(90-100\%) and accuracy (85-100\%) of determination of lymph node status; these outcomes are comparable to those of SN mapping for melanoma and breast cancer $[27,28]$. Recently, Wang et al. [27] reported a systematic review that evaluated the diagnostic value of $\mathrm{SN} \mathrm{bi-}$ opsy for gastric cancer. The results of their large-scale meta-analysis, which included 38 relevant studies with 2,128 patients, demonstrated that the $\mathrm{SN}$ detection rate and accuracy of prediction of lymph node metastasis based on SN status were 94 and 92\%, respectively [27]. They concluded that the SN concept is technically feasible for gastric cancer, especially cases with early $\mathrm{T}$ stage (T1), with the use of combined tracers and submucosal injection methods during the SN biopsy procedures.

A study group from the Japan Society of Sentinel Node Navigation Surgery (JSNNS) recently conducted a multicenter prospective trial of SN mapping using a dual-tracer method with a radioactive colloid and blue dye [29]. In the trial, SN mapping was performed between 2004 and 2008 for approximately 400 patients with early gastric cancer at 12 comprehensive hospitals, including our institution. Eligibility criteria were that patients had a cT1N0M0 or cT2N0M0 single tumor with the diameter of the primary lesion less than $4 \mathrm{~cm}$, without any previous treatments. The $\mathrm{SN}$ detection rate was $98 \%$ and the accuracy of determination of metastatic status was 99\% [29]. The results of that clinical trial are expected to provide us with perspectives on the future of SN navigation surgery for early gastric cancer.

In contrast, the Japan Clinical Oncology Group (JCOG) also conducted a multicenter prospective trial of SN biopsy for cT1N0 gastric cancer [30]. The JCOG 0302 study was designed to assess the feasibility and accuracy of SN biopsy by the dye-guided method with intraoperative subserosal direct injection of ICG. Although the details of the study have not yet been published, recruitment of patients for the JCOG 0302 study was terminated midway because of the high false-negative rate. Several factors were involved, including technical issues of the dye-only guided method with subserosal injection of the tracer and limited sensitivity of intraoperative histological diagnosis of SN status. One of the most critical limitations in the JCOG 0302 study might be the fact that there were only 5 initial cases for training in each institution. On the contrary, in our JSNNS study, 30 cases were required as the minimum experience for the initial learning phase to participate in the multicenter study based on previous reports for multicenter studies of SN biopsies for breast cancer [31]. 


\section{Future Direction of Laparoscopic SN Mapping for Early Gastric Cancer}

The distribution of sentinel lymphatic basins and the pathological status of SNs would be useful in deciding on the minimized extent of gastric resection and for avoiding the universal application of distal or total gastrectomy with D2 dissection. Appropriate indications for partial (wedge) resection, segmental gastrectomy, pylorus-preserving gastrectomy, and proximal gastrectomy for cT1N0 gastric cancer could be individually determined on the basis of SN status. Currently, surgical treatment of cT1N0 gastric cancer can be individualized on the basis of SN navigation [15-17, 19]. Various types of laparoscopic function-preserving surgeries are applicable for cancer patients with negative SNs for metastasis by intraoperative pathological diagnosis. For example, laparoscopic partial (wedge) resection of the stomach is applicable for patients with a sentinel lymphatic basin on the greater curvature side. Laparoscopic segmental gastrectomy with a pylorus-preserving procedure is feasible in patients with a primary lesion more than $4 \mathrm{~cm}$ from the pylorus ring and having two sentinel lymphatic basins on the lesser and greater curvature sides. Also, laparoscopyassisted proximal gastrectomy is an attractive alternative to total gastrectomy for patients with cT1N0 cancer of the upper third of the stomach. We previously reported the feasibility of laparoscopy-assisted proximal gastrectomy with SN mapping for 37 patients with cT1N0 proximal gastric cancer (fig. 3) [19]. The sensitivity of prediction of nodal metastasis and diagnostic accuracy based on SN status were both $100 \%$ ( $3 / 3$ and $37 / 37$, respectively) and all patients have been free from recurrence. Earlier recovery after surgery and preservation of QOL in the late phase can be achieved by laparoscopic limited gastrectomy with SN navigation.

Although the accuracy of determining lymph node status by laparoscopic SN mapping may vary between single institutional studies, these results are generally acceptable in cases with early gastric cancer patients [27]. Moreover, these reports have demonstrated that sentinel lymphatic basins contain truly positive nodes, even when the SN biopsy produces a false negative. Sentinel lymphatic basin dissection can provide an acceptable safety net for the clinical application of laparoscopic SN sampling for gastric cancer $[16,17]$. This novel surgical procedure may be a feasible and reliable option for cT1N0 early gastric cancer. Our study group in the JSNNS is currently preparing to conduct the multicenter phase III trial which will compare the function-preserving gastrec-

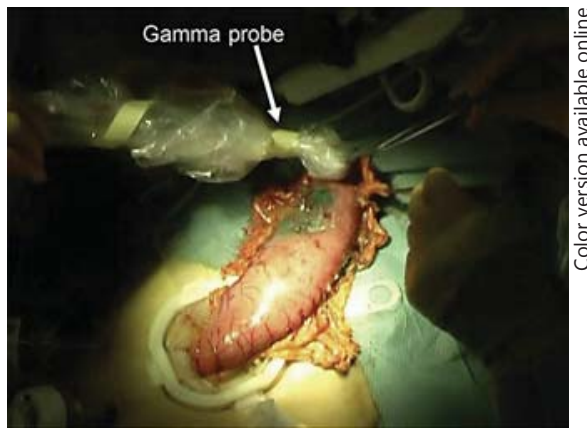

Fig. 3. Laparoscopy-assisted proximal gastrectomy with SN mapping. The stomach was pulled out through the small incisional wound. Intraoperative gamma probing was performed extracorporeally to identify SNs.

tomy with SN mapping to the conventional distal or total gastrectomy in terms of curability and patients' QOL as the next step.

The pattern of the sentinel lymphatic basin could be approximately estimated by primary tumor location according to the accumulation of the results of SN mapping in cT1N0 gastric cancer [19]. However, at the same time, the fact that the distribution of SNs shows an unpredictable pattern in some patients cannot be ignored. We believe that intraoperative SN mapping and biopsy are indispensable to verify that all SNs are negative for cancer metastasis and to decide the actual extent of the sentinel lymphatic basin if we attempt the laparoscopic functionpreserving gastrectomy because other metastatic lymph nodes are frequently located beyond the sentinel lymphatic basin when the $\mathrm{SN}$ are microscopically positive for metastasis.

To date, we have no other useful tools which can predict the number and the location of all metastatic nodes intraoperatively in patients with positive SNs. Moreover, the most recent Japanese guidelines recommend standard $\mathrm{D} 2$ lymphadenectomy in patients with $\mathrm{T} 1 \mathrm{~N} 1$ gastric cancer $[19,32]$. Therefore, distal or total gastrectomy with $\mathrm{D} 2$ dissection would be favorable as a more radical and curative lymphadenectomy for patients with positive $\mathrm{SN}$ at this time. Further studies will be needed to verify the appropriate extent of lymphadenectomy for patients with positive $\mathrm{SN}$.

A combination of laparoscopic SN biopsy and endoscopic mucosal resection (EMR)/endoscopic submucosal dissection (ESD) for early gastric cancer is another attractive option as a novel, whole stomach-preserving, minimally invasive approach. The indications of endo- 


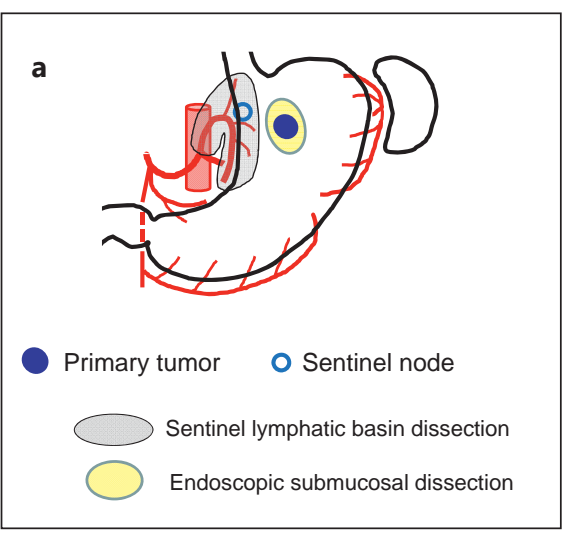

Fig. 4. Laparoscopic sentinel lymphatic basin dissection plus ESD. a Diagram of sentinel lymphatic basin dissection plus ESD. b Laparoscopic approach. c Sentinel lymphatic basin resection. d Sentinel lymphatic basin on the back table. The intensity of radioactiv-

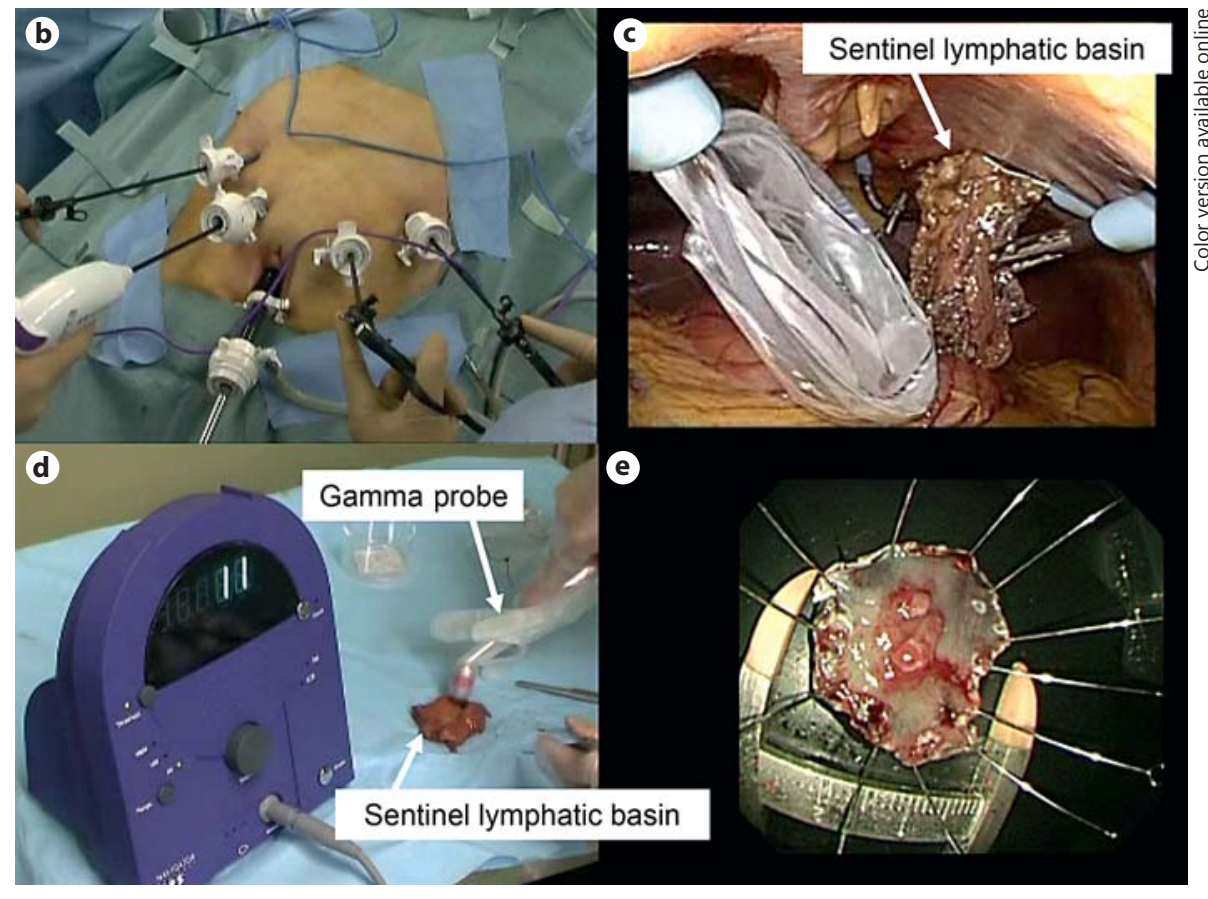

ity in the $\mathrm{SN}$ can be visualized by the scintillation counter. e ESD was subsequently performed in patients with negative SNs for metastasis by intraoperative pathological diagnosis. scopic treatments such as EMR are limited as follows: (1) mucosal tumors, (2) histologically differentiated-type adenocarcinomas, (3) less than $2-\mathrm{cm}$ diameter tumors, and (4) no ulcer or no ulcer scar in the lesion. This is because lymph node metastasis is considered absent if the primary tumor is within these criteria $[32,33]$. However, recent technical advances in endoscopic treatment have enabled the en bloc resection of much larger and slightly deeper early gastric cancers, including undifferentiated or ulcerated types, by ESD. If all SNs are pathologically negative for cancer metastasis, theoretically, EMR/ESD instead of gastrectomy may be sufficient for the curative resection of $\mathrm{CT} 1$ gastric cancer beyond the EMR criteria (fig. 4).

However, it is probably too soon for laparoscopic SN mapping and consequent intraoperative EMR/ESD for $\mathrm{SN}$-negative cases to become an accepted approach in clinical practice because we still have a number of technical controversies to be resolved. These include the accuracy of intraoperative pathological diagnosis, the necessity of full-thickness resection, and the possibility of cancer cells being present in afferent lymphatic vessels leading to SNs. Further studies are required to verify the safety and effectiveness of combined treatments involving laparoscopic SN biopsy and EMR/ESD.

Nowadays, laparoscopy-assisted distal gastrectomy or laparoscopy-assisted proximal gastrectomy is frequently applied to the patients with early gastric cancer according to the results of pathological assessments of primary tumors resected by EMR/ESD in clinical practices. To date, it has not been clarified whether SN mapping is feasible even after EMR/ESD. One of the most important issues is whether lymphatic flow from the primary tumor to the original SNs may change after EMR/ESD. In our preliminary study, however, at least the sentinel lymphatic basin is not markedly affected by previous EMR/ESD (data not shown). Modified gastrectomy according to SN distribution and metastatic status might be feasible even for the patients who underwent EMR/ESD prior to surgery.

The recent appearance of a new technique, referred to as natural orifice transluminal endoscopic surgery (NOTES), may be figuratively regarded as a second coming of the laparoscopic surgery of the 1990s, and currently there has been a drastic acceleration in the exploration of new techniques [34, 35]. Cahill et al. [36] reported on lymphatic mapping and SN biopsy by transgastric 
NOTES in a porcine model. The authors used the transgastric NOTES technique to easily perform lymphatic mapping and $\mathrm{SN}$ biopsy in the colonic mesentery. They concluded that SN biopsy is indeed feasible without an abdominal incision. Their article may represent a valuable signpost for the future course of SN biopsy for early gastric cancer. The combination of EMR/ESD with SN biopsy by NOTES may be an ideal minimally invasive surgical strategy for cN0 early gastric cancer [37].

\section{Conclusion}

For early-stage gastric cancer such as cT1N0, for which a better prognosis can be achieved through conventional surgical approaches, the establishment of individualized, minimally invasive treatments that may retain the patients' QOL should be the next surgical challenge. Although further studies are required for careful validation, EMR/ESD combined with SN navigation surgery could be a promising strategy to achieve this goal.

\section{References}

1 Morton DL, Wen DR, Wong JH, Economou JS, Cagle LA, Storm FK, Foshag LJ, Cochran AJ: Technical details of intraoperative lymphatic mapping for early stage melanoma. Arch Surg 1992;127:392-399.

2 Giuliano AE, Kirgan DM, Guenther JM, Morton DL: Lymphatic mapping and sentinel lymphadenectomy for breast cancer. Ann Surg 1994;220:391-401.

-3 Bilchik AJ, Saha S, Wiese D, Stonecypher JA, Wood TF, Sostrin S, Turner RR, Wang HJ, Morton DL, Hoon DS: Molecular staging of early colon cancer on the basis of sentinel node analysis: a multicenter phase II trial. J Clin Oncol 2001;19:1128-1136.

-4 Kitano S, Iso Y, Moriyama M, Sugimachi K: Laparoscopy-assisted Billroth I gastrectomy. Surg Laparosc Endosc 1994;4:146-148.

$\checkmark 5$ Adachi Y, Shiraishi N, Shiromizu A, Shiromizu A, Bandoh T, Aramaki M, Kitano S: Laparoscopy-assisted Billroth I gastrectomy compared with conventional open gastrectomy. Arch Surg 2000;135:806-810.

6 Shinohara T, Kanaya S, Taniguchi K, Fujita T, Yanaga K, Uyama I: Laparoscopic total gastrectomy with D2 lymph node dissection for gastric cancer. Arch Surg 2009;144:11381142.

7 Hur H, Jeon HM, Kim W: Laparoscopy-assisted distal gastrectomy with D2 lymphadenectomy for T2b advanced gastric cancers: three years' experience. J Surg Oncol 2008;98: 515-519.

8 Kim YW, Baik YH, Yun YH, Nam BH, Kim DH, Choi IJ, Bae JM: Improved quality of life outcomes after laparoscopy-assisted distal gastrectomy for early gastric cancer: results of a prospective randomized clinical trial. Ann Surg 2008;248:721-727.

-9 Kitagawa Y, Fujii H, Mukai M, Kubota T, Ando N, Watanabe M, Ohgami M, Otani Y, Ozawa S, Hasegawa H, Furukawa T, Kumai K, Ikeda T, Nakahara T, Kubo A, Kitajima M: The role of the sentinel lymph node in gastrointestinal cancer. Surg Clin N Am 2000;80: 1799-1809.
10 Kitagawa Y, Kitano S, Kubota T, Kumai K, Otani Y, Saikawa Y, Yoshida M, Kitajima M: Minimally invasive surgery for gastric cancer - toward a confluence of two major streams: a review. Gastric Cancer 2005;8:103-110.

-11 Kitagawa Y, Fujii H, Kumai K, Kubota T, Otani Y, Saikawa Y, Yoshida M, Kubo A, Kitajima M: Recent advances in sentinel node navigation for gastric cancer: a paradigm shift of surgical management. J Surg Oncol 2005; 90:147-152.

12 Kitagawa Y, Kitajima M: Diagnostic validity of radio-guided sentinel node mapping for gastric cancer: a review of current status and future direction. Surg Technol Int 2006;15: $32-36$.

13 Nakahara T, Kitagawa Y, Takeuchi H, Fujii H, Suzuki T, Mukai M, Kitajima M, Kubo A: Preoperative lymphoscintigraphy for detection of sentinel lymph node in patients with gastric cancer: initial experience. Ann Surg Oncol 2008;15:1447-1453.

14 Vermeeren L, van der Ploeg IM, Olmos RA, Meinhardt W, Klop WM, Kroon BB, Nieweg OE: SPECT/CT for preoperative sentinel node localization. J Surg Oncol 2010;101: 184-190.

15 Saikawa Y, Otani Y, Kitagawa Y, Yoshida M, Wada N, Kubota T, Kumai K, Sugino Y, Mukai M, Kameyama K, Kubo A, Kitajima M: Interim results of sentinel node biopsy during laparoscopic gastrectomy: possible role in function-preserving surgery for early cancer. World J Surg 2006;30:1962-1968.

16 Takeuchi H, Saikawa Y, Kitagawa Y: Laparoscopic sentinel node navigation surgery for early gastric cancer. Asian J Endosc Surg 2009;2:13-17.

17 Takeuchi H, Kitagawa Y: New sentinel node mapping technologies for early gastric cancer. Ann Surg Oncol 2013;20:522-532.

18 Kitagawa Y, Saikawa Y, Takeuchi H, Mukai M, Nakahara T, Kubo A, Kitajima M: Sentinel node navigation in early stage gastric cancer: updated data and current status. Scand J Surg 2006;95:256-259.
9 Takeuchi H, Oyama T, Kamiya S, Nakamura R, Takahashi T, Wada N, Saikawa Y, Kitagawa Y: Laparoscopy-assisted proximal gastrectomy with sentinel node mapping for early gastric cancer. World J Surg 2011;35:2463-2471.

20 Kinami S, Fujimura T, Ojima E, Fushida S, Ojima T, Funaki H, Fujita H, Takamura $\mathrm{H}$, Ninomiya I, Nishimura G, Kayahara M, Ohta T, Yoh Z: PTD classification: proposal for a new classification of gastric cancer location based on physiological lymphatic flow. Int J Clin Oncol 2008;13:320-329.

21 Tajima Y, Murakami M, Yamazaki K, Masuda Y, Kato M, Sato A, Goto S, Otsuka K, Kato T, Kusano M: Sentinel node mapping guided by indocyanine green fluorescence imaging during laparoscopic surgery in gastric cancer. Ann Surg Oncol 2010;17:1787-1793.

22 Ishikawa K, Yasuda K, Shiromizu T, Etoh T, Shiraishi N, Kitano S: Laparoscopic sentinel node navigation achieved by infrared ray electronic endoscopy system in patients with gastric cancer. Surg Endosc 2007;21:1131-1134.

23 Ohdaira H, Nimura H, Mitsumori N, Takahashi N, Kashiwagi H, Yanaga K: Validity of modified gastrectomy combined with sentinel node navigation surgery for early gastric cancer. Gastric Cancer 2007;10:117-122.

24 Nimura $\mathrm{H}$, Narimiya N, Mitsumori N, Yamazaki Y, Yanaga K, Urashima M: Infrared ray electronic endoscopy combined with indocyanine green injection for detection of sentinel nodes of patients with gastric cancer. Br J Surg 2004;91:575-579.

25 Miyashiro I, Miyoshi N, Hiratsuka M, Kishi K, Yamada T, Ohue M, Ohigashi H, Yano M, Ishikawa $\mathrm{O}$, Imaoka $\mathrm{S}$ : Detection of sentinel node in gastric cancer surgery by indocyanine green fluorescence imaging: comparison with infrared imaging. Ann Surg Oncol 2008; 15 : 1640-1643.

26 Tajima Y, Yamazaki K, Masuda Y, Kato M, Yasuda D, Aoki T, Kato T, Murakami M, Miwa M, Kusano M: Sentinel node mapping guided by indocyanine green fluorescence imaging in gastric cancer. Ann Surg 2009;249: 58-62. 
27 Wang Z, Dong ZY, Chen JQ, Liu JL: Diagnostic value of sentinel lymph node biopsy in gastric cancer: a meta-analysis. Ann Surg Oncol 2012;19:1541-1550.

-28 Can MF, Yagci G, Cetiner S: Sentinel lymph node biopsy for gastric cancer: where do we stand? World J Gastrointest Surg 2011;27: 131-137.

29 Kitagawa Y, Takeuchi H, Takagi Y, Natsugoe S, Terashima M, Murakami N, Fujimura T, Sakamoto J, Aikou T, Kitajima M: Prospective multicenter trial of sentinel node mapping for gastric cancer (abstract 4518). J Clin Oncol 2009;27(suppl):15S.
30 Miyashiro I: What is the problem in clinical application of sentinel node concept to gastric cancer surgery? J Gastric Cancer 2012;12:712.

31 Cox CE, Salud CJ, Cantor A, Bass SS, Peltz ES, Ebert MD, Nguyen K, Reintgen DS: Learning curves for breast cancer sentinel lymph node mapping based on surgical volume analysis. J Am Coll Surg 2001;193:593-600.

32 Japanese Gastric Cancer Association: Gastric Cancer Treatment Guidelines, ed 3. Tokyo, Kanehara Publishing Co., 2010.

33 Sano T, Hollowood K: Early gastric cancer: diagnosis and less invasive treatments. Scand J Surg 2006;95:249-255.

- 34 Kalloo AN, Singh VK, Jagannath SB, Niiyama H, Hill SL, Vaughn CA, Magee CA, Kantsevoy SV: Flexible transgastric peritoneoscopy: a novel approach to diagnostic and therapeutic interventions in the peritoneal cavity. Gastrointest Endosc 2004;60:114-117.
35 Allori AC, Leitman IM, Heitman E: Natural orifice transluminal endoscopic surgery. Arch Surg 2008;243:333-334.

-36 Cahill RA, Perretta S, Leroy J, Dallemagne B, Marescaux J: Lymphatic mapping and sentinel node biopsy in the colonic mesentery by natural orifice transluminal endoscopic surgery (NOTES). Ann Surg Oncol 2008;15: 2677-2683.

37 Takeuchi H, Kitagawa Y: Sentinel node biopsy without scars. Does natural orifice transluminal endoscopic surgery herald a new era for early GI cancer? Ann Surg Oncol 2008; 15 : 2639-2640. 